\title{
Efectos de un programa de gimnasia cerebral sobre la lectura crítica de los estudiantes admitidos en la universidad popular del Cesar
}

DOI: $10.46932 / \mathrm{sjjdv2n1-025}$

Received in: November 1st, 2020

Accepted in: December 30th, 2020

\author{
Dra. María Mercedes Colina \\ Dra. Ciencias Gerenciales, Docente Investigador de la Universidad Mariana. Jurado de tesis doctorales. \\ Dirección callejas del norte calle 4c\#23-05. \\ E-mail: merede3371@gmail.com
}

\section{Lesby Johanna Lora Carrillo}

Docente Investigador de la Universidad Popular del Cesar, Asesora del Semillero Educación y tecnología EDUTEC, Psicóloga Social, Esp. Métodos y técnicas de Investigación en Ciencias Sociales, Mg. Teoría y Práctica de la Prosocialidad y sus Aplicaciones en la Logoterapia. Perteneciente al grupo de Investigación Interdisciplinario Estudio Del Pensamiento Numérico, Políticas Públicas De Ciencia Y Tecnología, Producción Agraria, Medio Ambiente, Y Problemática De La Educación Latinoamericana Y Del Caribe. Dirección: Calle 4 No 19-45.

E-mail: lesbylora@unicesar.edu.co.

\section{Álvaro Arrieta Hernández}

Lic. en Lengua Castellana e inglés, Mg. en Lingüística, Docente Investigador Universidad Popular del Cesar, Dirección: Calle 7E \# 27 - 14 Villa Concha,

E-mail: alvaroarrieta@unicesar.edu.co

\section{RESUMEN}

La presente investigación tiene el objetivo determinar los efectos de un programa de gimnasia cerebral sobre la lectura crítica de los estudiantes admitidos en la Universidad Popular del Cesar, para lo cual se realizó una revisión d de textos y artículos indexados, considerando las posturas epistemológicas Pérez, (2014), Romero, (2014), Cely \& Sierra, (2011), López (2011), Baquerizo, (2013), entre otros. En este sentido, se pretende describir los niveles de comprensión de lectura crítica antes y después de la aplicación del programa de gimnasia cerebral para comparar los hallazgos, evaluando de los resultados en función de mejorar los niveles de lectura crítica. En cuanto a la metodología la investigación es de tipo experimental con nivel explicativo bajo la modalidad de experimento de campo, con un diseño cuasi experimental con pre y post prueba del grupo control y experimental. La población estará conformada por 82 estudiantes admitidos para el periodo II del año 2018 en la asignatura de fundamentos de psicología, del turno matutino cuyas edades comprenden entre los 17 a 19 años, bajo una muestra intencional, para la correspondencia del instrumento se requerirán 3 expertos y para su confiabilidad se utilizará el método de cálculo de Kuder Richardson. Con resultados esperados se pretende mostrar productos tangibles y medibles generando impacto en los procesos de aprendizaje de los estudiantes; para los docentes la aplicación de estrategias innovadoras que conlleven a mejorar los niveles de aprendizajes de lectura crítica, a través del programa de gimnasia cerebral.

Palabras claves. Programa de gimnasia cerebral, Lectura crítica, Universidad Popular del Cesar. 


\section{INTRODUCCIÓN}

En las últimas décadas, el mundo ha venido experimentando cambios en el ámbito educativo universitario, donde los procesos están cada día mas acelerados en cuestión de aprendizaje y requieren formas distintas de hacer las cosas para poderse adaptar a las exigencias del siglo XXI, dentro de estas turbulencias uno de los avances más relevantes para la lectura es la neurociencia.

En este orden, para Contreras (2015), dentro de los procesos educativos de enseñanza aprendizaje de la lectura, la neurociencia aporta el reconocimiento del funcionamiento del cerebro en el quehacer pedagógico, lo que permite el abordaje de temas a través de la utilización del cerebro triuno, inteligencia emocional, programación neurolingüística, gimnasia cerebral, entre otros, los cuales brindan estrategias que aminora la problemática.

Cabe destacar, que el aprendizaje de la lectura crítica y la inserción al mundo escrito hacen parte de una necesidad a nivel mundial. En este particular Whitehurst y Lonigan (2010), afirman que de cada tres estudiantes universitarios, uno experimenta dificultades significativas para leer, por tanto no alcanza el nivel de dominio esperado en los plazos previstos, evidenciándose la raíz del problema en el cómo el docente produce el acercamiento a la lectura, para crear una relación placentera entre el libro y el lector, y que se mantenga una conexión de bienestar personal sin perder el encanto y la experiencia positiva que deja el texto.

Ahora bien, cuando el sistema universitario permite conocer nuevos escenarios, nacen estructuras innovadoras para construcción de la enseñanza generando un impacto en el desarrollo académico del estudiante ya que direcciona las competencias procedimentales, actitudinales y cognitivas efectivamente, empoderando a los estudiantes de sus habilidades, a través de la utilización de todo el cerebro.

De manera, que la aplicabilidad de la neurociencia desde la gimnasia cerebral, permite desplegar actividades del sistema cognitivo para el aprendizaje de la lectura crítica y desarrollo de habilidades, estimulando procesos como la atención, percepción, pensamiento, lenguaje, imaginación, creatividad y memoria para que sea agradable y significativo. Goswami (2006)

En torno al tema, la gimnasia cerebral como programa con estrategias sensorio motoras y técnicas generan conexiones neuronales a través de ejercicios corporales y oculares manteniendo la atención del estudiante, durante un tiempo prolongado en las actividades, alineando el funcionamiento del hemisferio izquierdo y el derecho propiciando el desarrollo de la creatividad, alcance de metas, Ibarra (2009); lo cual fortalece aprendizaje, ya que su clave se fundamenta en desbloquear los procesos cognitivos para poder procesar la información de la mejor manera.

Cabe destacar, que en la Educación Superior a nivel latinoamericano se han detectado casos de analfabetismo funcional, el cual va más allá de la simple sub - utilización de las destrezas adquiridas 
durante años anteriores, situación que se evidencia por el bajo nivel de comprensión de la lectura crítica del estudiante, lo cual repercute a la hora de identificar las ideas principales expuestas en un texto, captar la intención del escritor y en la redacción coherente de resúmenes de los textos objeto de lectura

Al respecto, Jiménez (2008), indica que la gimnasia cerebral como una práctica física, optimiza el funcionamiento del cerebro y genera neurotransmisores como la noradrenalina y la dopamina que intervienen en los procesos de atención preparando a los estudiantes para tener mejor comprensión de la lectura y memoria aprendiendo eficazmente y de manera creativa Huerta (2008).

En este sentido, ha de considerarse la lectura crítica como proceso cognitivo, resaltando el papel del desarrollo de los tres niveles de comprensión: literal, inferencial y crítica, de tal manera que se desarrolle paralelamente las habilidades básicas de interpretación, para formarse una opinión, sacar ideas centrales y extraer conclusiones; organización, estableciendo secuencias del texto que le permitan discernir sobre el mismo; por ultimo valorar las relaciones causales diferenciando lo verdadero de lo falso, captando el sentido de la interpretación. Gail (2003)

Considerando las implicaciones anteriores, desde la perspectiva de Freire (2013), la lectura, no es simplemente trasladar el material escrito a la lengua oral, eso sería una simple técnica de decodificación, leer significa interactuar con un texto, comprenderlo y utilizarlo con fines específicos, llevarlo a la vida práctica. Por ende, un estudiante universitario que no tenga la capacidad de descifrar el lenguaje se le hará cuesta arriba adquirir el conocimiento.

Colombia no escapa de esta realidad, a pesar que los institutos escolares de básica secundaria preparan a los estudiantes para presentar las pruebas ICFES y dentro de sus lineamientos está inmerso el desarrollo de la comprensión lectora; los planes de estudio que se promueven son dependiente de la necesidad real del estudiante; aun cuando los docentes tiene conocimientos conceptuales disciplinarios, carecen de herramientas o instrumentos aplicables para el desarrollo de habilidades que conduzcan a enfrentar los retos propuestos por el sistema universitario, escenario limita el proceso de enseñanza convirtiéndose en un obstáculo para avanzar.

En este contexto Rodríguez (2013), afirma que los niveles de comprensión lectora de los estudiantes que ingresan al sistema universitarios colombiano se encuentran por debajo de lo esperado, ya que carecen de estrategias que les permitan inferir en detalles de las lecturas, secuencias del texto, así como en las relaciones de causa y efecto, realizando conjeturas incorrectas; lo cual conlleva a frustraciones y baja calidad académica.

Cabe destacar, que la lectura crítica va más allá de la comprensión de lo que el autor dice, por tanto, se busca un nivel donde el estudiante sea crítico y evalué cuidadosamente lo que se presenta 
haciéndose cuestionamientos que le permitan llegar a una conclusión razonable, además de mantener la atención y concentración en el texto, encontrando su motivación para seguir aprendiendo. Aguirre. (2008).

Ahora bien, La universidad Popular del Cesar, inmersa en este problema ingresa nuevos estudiantes en cada periodo académico, algunos con dificultades de aprendizaje, para los cuales no existe una prueba de selección; situación que lleva a los docentes presentar quejas continuas sobre el desenvolvimiento académico de los mismos atendiendo a sus exigencias particulares sobre las asignaturas, manifestando que los procesos cognitivos están por debajo de lo esperado, razón por la cual se les dificulta leer, escribir, analizar e interpretar, esta situación hace que la enseñanza en el salón de clase tenga que retroceder a estadios anteriores para poder avanzar.

Visto desde estas perspectivas, es de relevancia para la universidad Popular del Cesar establecer mecanismos de fortalecimiento para mejorar la calidad educativa de los estudiantes de manera integral, conectándolos con el mundo de la lectura atendiendo sus intereses, de tal manera que se pueda dar respuesta a los docentes y aumentar los indicadores de rendimiento exigidos por el Ministerio de Educación Nacional.

De allí que uno de los beneficios que subyace en la aplicación de un programa de gimnasia cerebral que fortalezca las habilidades de la lectura crítica; enmarcado en avances significativos de comprensión, análisis y aplicación de la misma bajo una perspectiva pedagógica innovadora que desarrolla las estructuras y procesos mentales con ejercicios que intervienen en la elaboración y construcción del conocimiento, actuando como espiral en crecimiento de la práctica del aprendizaje, haciéndola más atractiva para sus intereses.

Este escenario, conlleva al paradigma de la nueva ciencia sobre los procesos de innovación, concibiendo el sistema educativo como abierto, sinérgico e interdependiente, que genera cambios a partir de cada situación y coadyuva en el continuo de aprendizaje integral de los estudiantes

Siendo conocido los argumentos que soportan esta investigación, constituye una herramienta importante para la formación de los nuevos estudiantes de la Universidad Popular del Cesar, ya que proporciona referentes sobre los efectos de un programa de gimnasia cerebral en la lectura crítica, potencializando el quehacer pedagógico así como las relaciones de los actores participantes en el proceso de enseñanza, fortaleciendo el rendimiento académico desde el inicio de su carrera, además conjuga en los lineamientos del Ministerio de Educación Superior (2013) y con el proyecto institucional cognitivo contextual, constructivista de la institución, con responsabilidad social frente al municipio, la nación y el país .

Por otro lado, los resultados servirán como soporte significativo para fomentar hábitos y el gusto por la lectura, ofreciendo información relevante para emprender cambios significativos al interior de la 
institución educativa, construyendo nuevas formas de aprendizaje que permita el desarrollo de habilidades cónsonas a las exigencias del entorno; formulando acciones donde los docentes y los estudiantes contrarresten el analfabetismo funcional.

En este orden de ideas, al no dar solución a los problemas de lectura crítica en los estudiantes admitidos en la Universidad Popular del Cesar a corto plazo se observarían un gran porcentaje de repitientes, bajo rendimiento académico, a mediano plazo problemas socioemocionales con carencia de hábitos de atención y largo plazo profesionales que no cumplen con las exigencias del entorno. Por tanto, sería conveniente trabajar en función de integrar los ejercicios de gimnasia cerebral para mejorar la lectura crítica.

En consecuencia, se hace necesario e imprescindible, determinar los efectos de un programa de gimnasia cerebral, que permita el logro de un aprendizaje significativo, facilitando la participación de los estudiantes que ingresan al sistema universitario como investigadores creativos, analíticos y reflexivos, de manera que, fijen en la estructura cognitiva, la imagen gráfica de las palabras de uso frecuente, interpretar, parafrasear la información, fortalezcan y enriquezcan el idioma; permitiendo promover un aprendizaje práctico, fácil y eficaz. Los planteamientos anteriores permiten formular la siguiente interrogante ¿Cuál es el efecto de un programa de gimnasia cerebral sobre la lectura crítica en estudiantes admitidos en la Universidad popular del Cesar?

Para llevar a cabo esta investigación se plantearon los siguientes objetivos, a nivel general determinar los efectos de un programa de gimnasia cerebral sobre la lectura crítica de los estudiantes admitidos en la Universidad Popular del Cesar y como específicos se consideran: Describir los niveles de comprensión de lectura crítica antes de la aplicación del programa. describir los niveles de comprensión de lectura crítica después de la aplicación del programa y por ultimo comparar ambos hallazgos para la evaluación de los resultados.

Para el desarrollo de los objeticos se tomaron como antecedentes investigaciones dirigidas al tema que otorgaron soporte al proyecto entre las cuales se resaltan: Pérez, (2014), Romero, (2014), González (2008), de igual forma se consideraron autores para la variable de lectura crítica Cely \& Sierra, (2011), Baquerizo, (2013), entre otros, que han escrito sobre el tema, evidenciando su impacto en el currículo, desde los campos de formación docente, acompañamiento estudiantil e investigación, promoviendo redes internas en torno al desarrollo de la lectura crítica, con espacios de socialización de experiencias y capacitación en las distintas facultades y programas de forma presencial, favorecedores de las habilidades en comprensión y producción de textos propios de las disciplinas.

Con base a lo anteriormente y considerando las posturas de autores expertos en el tema se sustentan las variables propuestas para la realización del proyecto las cuales se describen a continuación. 
Programa de Gimnasia cerebral. Para López (2011), un programa es un instrumento rector que se origina partiendo de la evaluación de la situación presentada, se rige por una planificación para cumplir las metas propuestas, de tal manera que los resultados se pueden medir en términos cuantificables, con tiempo y espacios definidos.

En este sentido, el programa de gimnasia cerebral constituye ejercicios con movimientos físicos que interconectan los hemisferios cerebrales, desarrollando conexiones neuronales esenciales para el aprendizaje de lectura, atención, concentración, relaciones interpersonales, entre otros. Benavides (2014). Por ende, en el proceso de la lectura crítica permite que el estudiante logre integrar la percepción, producción corporal y mental, adentrándose a estrategias que permiten ver, hacer y producir, escenario que apoya la acción pedagógica elevando los niveles de rendimiento.

No obstante, Sambrano (2014) define gimnasia cerebral como un sistema de estrategias que involucran la mente y el cuerpo mejorando los procesos del pensamiento, basándose en el principio que no hay aprendizaje sin movimiento, con su práctica se ejercitan las conexiones neuronales permitiéndole al estudiante ser más creativo.

De allí que, una vez puesta en práctica las estrategias de la gimnasia cerebral para obtener mayor rendimiento en la lectura crítica de los estudiantes, podrán resolver los problemas presentados en el aula de una manera más rápida y eficaz, atendiendo a sus necesidades, así como las exigencias del entorno educativo.

Estrategias de la gimnasia cerebral. Dennison (1997), afirma que se fundamentan en ejercicios de movimientos corporales, que logran modificar los sentimientos, pensamientos, generando nuevas formas de ver las cosas, por ende, ofrecen herramientas que permiten instaurar la confianza del estudiante apropiándose de sus habilidades, facilitando la construcción de redes neuronales. Portillo (2008)

En el caso de los estudiantes universitarios, al activarse las neuronas se incrementa la velocidad en la transmisión de los impulsos nerviosos, además protege y asiste la regeneración de los nervios cuando han sido dañados, propiciando el mejoramiento del aprendizaje.

Ibarra (2009), clasifica las estrategias de gimnasia cerebral en tres (3) categorías: De línea central; obedecen al aumento en la coordinación de la mente y el cuerpo, constituye ocho (8) movimientos: marcha cruzada, respiración abdominal, ocho perezoso, mecedora, doble garabato, marcha cruzada en el suelo y el elefante. De estiramiento persigue reeducar al cuerpo para el cambio de posturas con seis (6) movimientos: activación del brazo, el búho, flexión de pie, toma a tierra, bombeo de la pantorrilla, balanceo de gravedad. y De energía, con siete (7) ejercicios: botones de equilibrio, del cerebro, de tierra Botones, espacio, agua y bostezo energético, todos orientados a facilitar el flujo de energía 
electromagnéticas a través del cuerpo con tareas que demandan del cerebro altos índices de actividad mental.

Bajo este escenario, estas estrategias, permite la permeabilidad de nuevos aprendizajes de manera sencilla, de allí que se hace necesario practicarlos como antesala de la lectura crítica de tal manera que el estudiante que inicia el periodo universitario enriquezca sus procesos cognitivos de forma natural, además de recibir estimulaciones para el aumento de su motivación hacia la lectura y rendimiento académico

\subsection{LECTURA CRÍTICA.}

Para Cely \& Sierra, (2011), parte de una interpretación, que se origina de la comprensión del texto, requiere de la transferencia hacia las experiencias, permitiendo al estudiante adquirir la capacidad de tomar una postura sobre lo que está plasmado en el papel, edificando sus conocimientos. Baquerizo, (2013), supone, que partiendo de la critica el lector es capaz de tomar una postura frente a lo que lee, con un fundamento propio.

Para tal efecto, atendiendo que los contextos universitarios exigen formas más elaboradas y complejas de la comprensión lectora, los estudiantes universitarios requieren nuevas formas de pensar, indagar, adquirir conocimientos, comprender, interpretar e interactuar con los textos, por tanto, deben desarrollar niveles óptimos que les permitan alcanzar las metas propuestas y un rendimiento académico consonó a las exigencias de la universidad y su profesión. En este orden, la lectura como fenómeno complejo de aprendizaje requiere de una planificación, estructura y objetivos claros que permitan la actualización durante su ejecución. Colom (2008)

En consecuencia, atender la responsabilidad del docente desde la gimnasia cerebral como antesala a la lectura para desarrollar las competencias de comprensión crítica desde los inicios de la etapa universitaria propicia el pensamiento lógico y creativo del estudiante en función de sus experiencias, también fortalece las estrategias pedagógicas utilizadas para enseñanza. Barriga. (2003)

Cabe destacar, que el ICFES, en su programa Saber Pro, (2013), institucionaliza y conceptúa elementos de seguimiento y cumplimiento en la metodología de evaluación que fortalecen la lectura crítica, considerando las competencias que tiene el lector de realizar un análisis profundo para dar cuenta de las relaciones entre los discursos y las prácticas socioculturales que las involucran condicionando al estudiante; donde el estudiante tiene a su disposición ejercicios de manera previa para reconstruir el sentido del texto reconociendo el contexto en el que se produce con sus condiciones discursivas. Desde este escenario se consideran los niveles de comprensión de la lectura crítica.

Niveles de comprensión de la lectura crítica. Serrano (2008) afirma que son las etapas por donde pasa el estudiante, que le permiten comprender e interpretar un texto exige estar en permanente 
movimiento, y desplazarse entre ciertas dimensiones, para poder decodificar, reconocer palabras, frases, relacionándolas con el significado. entre ellas se encuentran:

Textual evidente., comprende el contenido del texto a través de los procesos de ubicación y articulación de información, realizando una primera tarea cognitiva de apropiación literal o inferencial, con el propósito de conocer cuál es su contenido superficial y su significado global, se requiere comenzar con temas de interés en forma de resumen, evalúa ubicar la información y articular las piezas del texto.

Relacional intertextual. Obedece al reconocimiento de las relaciones que pueden ser identificadas al interior del texto o entre dos, se presentan en orden sintáctico o semántico, determina la disposición que tiene el estudiante para relacionar las piezas textuales; exige la indagación para relacionar los párrafos y darle sentido global, identificando las relaciones entre las ideas que subyacen en el mismo, evalúa el reconocimiento de las partes y la relación con otros textos.

Dimensión enunciativa. Ahonda las implicaciones del hecho comunicativo, y su relación con quiénes participan en la situación de comunicación, dependen de una audiencia específica, enfatiza la profundidad del texto, y la capacidad del estudiante para identificar situaciones discursivas en las que participan activamente; evalúa el uso condicionado del código (modalidad del enunciado), la caracterización de quiénes participan en dicha situación, identificación del propósito comunicativo y la intención que tiene el enunciador de acuerdo con la audiencia a la que se dirija.

Dimensión valorativa. Busca, lo que se encuentra detrás de lo escrito, exige del estudiante la capacidad para identificar los elementos ideológicos que subyacen al texto y que se han venido enmascarando con estrategias textuales, distingue el conocimiento en los aspectos sintácticos y semánticos en función de la reconstrucción del contexto en el que se produce e interpreta dicho texto, evalúa el desempeño cuando se reconoce los contenidos valorativos o ideológicos en los enunciados en un texto o situación de comunicación, también las perspectivas y puntos de vista que circulan.

Dimensión sociocultural. Reconoce la intención y el propósito que guían la puesta en la escena social de circulación del texto; tanto el hecho de la lectura como el de la producción, son prácticas que se actualizan bajo las circunstancias históricas, sociales e ideológicas donde se evalúa el desempeño del estudiante a través del reconocimiento del cómo las estrategias discursivas inciden sobre la audiencia y cómo los discursos configuran prácticas socioculturales.

En consideración a lo expuesto, el programa de gimnasia cerebral, fortalece a través de sus ejercicios la comprensión de los niveles de lectura crítica, estimulando los procesos cognitivos del estudiante a través de los ejercicios propuestos en función de alcanzar un aprendizaje significativo. 


\subsection{SISTEMA DE HIPÓTESIS}

\subsubsection{Hipótesis de la investigación}

Si se aplica un programa de gimnasia cerebral en los estudiantes admitidos en la Universidad Popular del Cesar, cursantes de la asignatura fundamentos de psicología, entonces su lectura crítica mejorara.

\subsection{HIPÓTESIS NULA}

Si se aplica un programa de gimnasia cerebral en los estudiantes admitidos en la Universidad Popular del Cesar, cursantes de la asignatura fundamentos de psicología, entonces su lectura crítica se mantendrá igual.

CUADRO 1. OPERACIONALIZACION DE LA VARIABLE

\begin{tabular}{|l|l|l|l|}
\hline \multicolumn{1}{|c|}{$\begin{array}{c}\text { Variable } \\
\text { independiente }\end{array}$} & Dimensiones & Subdimensiones & \multicolumn{1}{c|}{ autores } \\
\hline $\begin{array}{l}\text { Programa de } \\
\text { gimnasia cerebral }\end{array}$ & $\begin{array}{l}\text { Estrategias de } \\
\text { gimnasia cerebral }\end{array}$ & $\begin{array}{l}\text { De línea central } \\
\text { De estiramiento } \\
\text { Para reeducar el } \\
\text { cuerpo } \\
\text { De energía }\end{array}$ & $\begin{array}{l}\text { González (2008), } \\
\text { Dennison (2003), } \\
\text { Ibarra (2007) }\end{array}$ \\
\hline $\begin{array}{l}\text { Variable } \\
\text { dependiente }\end{array}$ & $\begin{array}{l}\text { Textual evidente. } \\
\text { Relacional } \\
\text { Lectura critica } \\
\text { comprensión de } \\
\text { la lectura critica }\end{array}$ & $\begin{array}{l}\text { Dimensión } \\
\text { enunciativa } \\
\text { Dimensión } \\
\text { valorativa } \\
\text { Dimensión } \\
\text { sociocultural }\end{array}$ & $\begin{array}{l}\text { Cely } \\
\text { Baquerizo, } \\
\text { (2013), ICFES, en } \\
\text { su programa Saber } \\
\text { Pro, (2013), }\end{array}$ \\
& & & \\
\hline
\end{tabular}

Fuente Elaboración propia (2018)

\section{METODOLOGÍA}

La investigación es de tipo experimental con nivel explicativo, ya que tiene como propósito establecer la relación de causa efecto existente entre la gimnasia cerebral y la lectura crítica, donde al aplicar el programa, ayudara el proceso de los estudiantes de la asignatura fundamentos de psicología que ingresan por primera vez a la universidad Por lo tanto, se llevó a cabo bajo la modalidad de experimento de campo manipulando la variable dependiente donde los estudiantes se desenvuelven de manera cotidiana, en este caso en la Universidad popular del cesar. Así mismo se constituye como una investigación de campo con un análisis sistemático de la realidad entendiendo su naturaleza y sus factores constituyentes. 
En cuanto al diseño, es cuasiexperimental ya que no hay asignación al azar o emparejamiento, apoyado con un grupo que no fue aleatorio, manipulando la variable independiente (gimnasia cerebral) para observar y medir los cambios que ocurren en la dependiente (lectura crítica) al tiempo que se controlan los factores intervinientes que puedan afectar el proceso.

Para realizar este estudio se pretende la utilización de un diseño con preprueba y postprueba en un solo grupo al que se le administra el programa una vez medida la variable para observar el nivel del mismo, cuyos resultados deben observarse con objetividad obteniendo conclusiones seguras para determinar el efecto del programa.

\section{Diagrama 1}

Diseño se presenta a través de la siguiente formula

\begin{tabular}{|c|c|c|c|}
\hline G1 & 01 & $\mathrm{X}$ & 02 \\
\hline $\mathrm{G} 2$ & 03 & ------- & 04 \\
\hline
\end{tabular}

\footnotetext{
Donde: G1. Grupo experimental

01 Preprueba

02 Postprueba

$\mathrm{X}$ el programa (gimnasia cereblar)

G2 Grupo control

03 Preprueba (grupo control)

02 Postprueba (grupo control)
}

Posteriormente, se realizará una postprueba a ambos grupos para determinar el efecto de una sobre la otra. Los criterios que se tomaron en cuenta para seleccionar los grupos fueron establecidos por: nuevos ingresos de la universidad, homogeneidad en ambos grupos, estudiantes de fundamentos de psicología, intervalos de tiempo entre la aplicación de una prueba y otra, y los ítems se aplicaran en forma aleatoria; con el fin de tener mayor validez en el diseño.

La población, estará constituida por 82 estudiantes admitidos para el periodo II del año 2018 la asignatura de fundamentos de psicología, del turno matutino cuyas edades comprenden entre los 17 hasta los 19 años de la Universidad Popular del Cesar. Bajo una muestra intencional, ambos grupos intactos escogidos por la accesibilidad del investigador.

\section{DEFINICIÓN DE LAS VARIABLES}

\subsection{VARIABLE INDEPENDIENTE}

Programa de gimnasia cerebral: Se manipulo a través de la presencia y ausencia del programa, el cual se estructuro de la siguiente manera: para el nivel de comprensión de lectura crítica ocho (8) ejercicios de línea central marcha cruzada, respiración abdominal, ocho perezoso, mecedora, doble garabato, marcha cruzada en el suelo y el elefante; para el nivel literal seis (6) movimientos de estiramiento 
activación del brazo, el búho, flexión de pie, toma a tierra, bombeo de la pantorrilla, balanceo de gravedad y para el inferencial siete (7) ejercicios de energía: botones de equilibrio, del cerebro, de tierra Botones, espacio, agua y bostezo energético, a través de 1 (sesión) por clase diseñado por las investigadoras durante doce (12) semanas, considerando las necesidades del grupo experimental

\subsection{VARIABLE DEPENDIENTE}

Lectura crítica. Será medida por los indicadores de la dimensión comprensión Literal, Inferencial y Crítica, a través de un baremo de interpretación como instrumento. La técnica de recolección de datos, abarca la descripción del instrumento, recolección de datos y sus propiedades psicométricas, el mismo se realizará con una serie de lectura con varias alternativas para escoger la apropiada y así poder realizar la medición de ambos grupos.

Mientras que, para el grupo experimental se realizará una serie de ejercicios basados en la gimnasia cerebral, durante un periodo de 12 doce semanas con frecuencia de dos (2) horas semanales en el grupo de fundamentos de psicología del primer semestre, el grupo control conformado por otra sección de fundamentos con las mismas características sin recibir tratamiento.

El instrumento estará conformado por veintisiete (27) reactivos los cuales indican el comportamiento de la variable lectura crítica, para lo cual se elaborara una escala de medición representada

De $0 \%$ a $20 \%$ muy baja comprensión, donde la dificultad de la prueba es mayor y su comprensión es casi nula.

De $21 \%$ al $35 \%$ baja comprensión, siendo esta deficiente, necesitando más esfuerzos para su interpretación

De $36 \%$ al 50\% mediana comprensión, siendo medianamente global, con ciertas limitaciones.

De $51 \%$ al $65 \%$ alta comprensión, comprenden con relativa facilidad el contenido global del material adaptado a su nivel de instrucción

Más del 66\% muy alta comprensión, alcanzando este nivel una vez que comprendan las lecturas sin realizar esfuerzos mayores, captando la esencia con facilidad.

De allí que para obtener el porcentaje se realizara la siguiente formula:

\section{$\underline{\mathrm{N}^{\circ} \text { de ítems acertados } \mathrm{x} 100=\%}$}

$\mathrm{N}^{\circ}$ total de ítems 
Para la variable gimnasia cerebral, se desarrollará el programa diseñado por Ibarra (2007), estructurando los ejercicios con la lectura critica

\subsection{PROPIEDADES PSICOMÉTRICAS.}

Para la correspondencia del instrumento con su contexto teórico, se determinará si el instrumento mide las necesidades presentadas en el proyecto; para lo cual se requiere el juicio de tres (3) expertos. Para su confiabilidad se utilizará el método de cálculo de Kuder Richardson ya que se presenta por escalas dicotómicas, cuyas alternativas son correctas o incorrectas y para el análisis de datos se considerará el programa estadístico SPSS 10.

\section{IMPACTO, PERTINENCIA Y RESULTADOS ESPERADOS:}

La investigación resultante ofrecera productos tangibles y medibles en el tiempo que generan un impacto positivo en los procesos de aprendizaje de la comunidad universitaria global y más específicamente en los estudiantes de fundamentos de psicología de la Universidad Popular del Cesar, por cuanto permite en líneas generales desarrollar estrategias que conllevan a mejorar los niveles de aprendizajes de lectura crítica, a través del programa de gimnasia cerebral, así como también estrategias para el fortalecimiento de la planificación del contenido impartido en las aulas; todo ello orientado a elevar el índice académico durante el periodo de la formación. Mención aparte, los resultados obtenidos servirán de base para otros programas de la institución, así como de otras universidades con características similares.

Igualmente, luego de su aplicación se pretende generar experiencias de aprendizajes significativos en el quehacer pedagógico de manera que los docentes sensibilicen su modo de enseñanza en función de nuevas formas para impactar el conocimiento, considerando la normativa que rige la educación en Colombia en el ámbito de desarrollo de competencias, así como el modelo pedagógico cognitivo contextual constructivista de la UPC 


\section{REFERENCIAS BIBLIOGRÁFICAS}

Aguirre De Ramírez, R. (2008). Fomentar la lectura y la escritura en estudiantes de formación docente. Acción pedagógica.

Arias (2006). El proyecto de Investigación. Introducción a la metodología científica. Caracas. Venezuela. Editorial Existeme.

Baquerizo, C. (2013). Aplicación de lectura crítica en los procesos de enseñanza aprendizaje para los estudiantes de segundo año especialización lengua y literatura Facultad de Filosofía de la Universidad de Guayaquil.

Barriga Arceo, F. D. (2003). Cognición situada y estrategias para el aprendizaje significativo

Benavides y Sierra, (2014). Estrategias didácticas para fomentar la lectura crítica desde la perspectiva de la transversalidad en la Universidad EAN, Colombia

Campbell (1996). Diseños experimentales, cuasiexperimentales en la investigacion social. Buenos aires. Cely, A \& Cierra, G. (2011). Lectura Crítica, creativa e investigativa para el desarrollo de las competencias comunicativas, cognitivas e investigativas en la educación superior. Bogotá: Universidad EAN.

Chávez (2007). Introducción a la investigación educativa. Artes gráficas tercera edición. Caracas

Colom Cañella, A. J. y Touriñán López, J. M. (2008). La Lectura en el siglo XXI. En XXVI Seminario Interuniversitario de Teoría de la Educación. Lectura y Educación. Universitat Autònoma de Barcelona: Barcelona, España

Contreras González, K. (2015). Una propuesta para mejorar la implementación del PNL en la zona escolar. Tesis de Especialización en Promoción de la Lectura de la Universidad Veracruzana.

Cannison P. (1997). Brain Gym. Aprendizaje con todo el cuerpo. España. Editorial Robin.

Ferrer, Gabriel (2002). Los hemisferios cerebrales y la inteligencia. Editorial Prentice Hall. México.

Freire (2013), lectura y competencia lectora. Revista de Educación vol.36, núm. 2. Universidad pontificea.

Gail (2003).Dennison, Maurice (2003). Perspectiva de la gimnasia cerebral. Editorial Mc Graw-Hill. Primera Edición. México.

Goswami U (2006) Neuroscience and education: from research to practice?. Nature 7

Hernández Y Batista (2006). Metodologia de la Investigacion. McGraw Hill. Mexico

Hernández, G. (2006). Estudio sobre los modelos conceptuales y la autorregulación de la comprensión y la composición de textos: Un análisis de sus posibles relaciones. Tesis doctoral, Facultad de Psicología, UNAM.

Huerta, Pedro (2000). La educación creativa en el mundo actual. La actividad expresiva. Pedagogía. Madrid-España. (pp. 107-227). 
Hurtado, J. (2001). El proyecto de investigación. Fundación Sypal - Editorial Magisterio. Bogotá.

Ibarra (2009) Aprende mejor con la gimnasia cerebral. Sexta edición. Mexico. Garnik. Ediciones P31

Jiménez, J. E. y O'shanahan Juan, I. (2008). Enseñanza de la lectura: de la teoría y la investigación a la práctica educativa. Revista Iberoamericana en Educación

López Acosta, S. A. (2015). Promoción de la lectura a través del sitio de lectores de la Universidad Veracruzana. Tesis de Especialización en Promoción de la Lectura de la Universidad Veracruzana

Mauricio (2008). La gimnasia cerebral en la educación. Editorial Tierra Nueva. Brasilia-Brasil.

Ministerio de educación. (2013), ICFES, en su programa Saber Pro.

Palella y Martins (2010); Metodología de la investigación cuantitativa” 3ra Edición. Fedupel. Caracas. Parra (2000). Estrategias didácticas. Facultad de Educación. Universidad de Costa Rica.

Pérez, (2014), Lectura crítica en la educación superior: una propuesta para el profesorado universitario.

Portillo (2008). Efectos de la gimnasia cerebral en la comprensión lectora de niños de segunda etapa de básica. Universidad Rafael Urdaneta. Venezuela.

Rodríguez, A., Solano, E., Martínez, A., \& Del Villar, L. (2013). "Lectura, escritura y calidad en la universidad colombiana"

Romero, Cueva y Barboza (2014), Gimnasia cerebral como estrategia para el desarrollo de la creatividad en los estudiantes de la UNERMB.Venezuela

Sambrano, J. (2014). Manual de uso. Caracas. Editorial Alfa.

Serrano de Moreno, M. S. (2008). El desarrollo de la comprensión crítica en los estudiantes universitarios: hacia una propuesta didáctica. Educere: artículo arbitrado.

Whitehurst y Lonigan (2010), Emergent Literacy: Development from Prereaders to Readers. Neuman, S. \& Dicknson, D.(Eds.) Handbook of Early Literacy 